\title{
Characteristics and outcomes of coronavirus disease 2019 (COVID-19) patients with cancer: A single-center retrospective observational study in Tokyo, Japan
}

\section{Shohei Nakamura}

Department of Medical Oncology, Tokyo Metropolitan Cancer and Infectious Diseases Center Komagome Hospital, Tokyo, Japan

Yusuke Kanemasa ( $\nabla$ y-kanemasa@cick.jp )

Department of Medical Oncology, Tokyo Metropolitan Cancer and Infectious Diseases Center Komagome Hospital, Tokyo, Japan https://orcid.org/0000-0002-9809-2008

\section{Yuya Atsuta}

Division of Hematology, Tokyo Metropolitan Cancer and Infectious Diseases Center Komagome Hospital, Tokyo, Japan

\section{Sho Fujiwara}

Department of Infectious Diseases, Tokyo Metropolitan Cancer and Infectious Diseases Center Komagome Hospital, Tokyo, Japan

\section{Masaru Tanaka}

Department of Infectious Diseases, Tokyo Metropolitan Cancer and Infectious Diseases Center Komagome Hospital, Tokyo, Japan

\section{Kazuaki Fukushima}

Department of Infectious Diseases, Tokyo Metropolitan Cancer and Infectious Diseases Center Komagome Hospital, Tokyo, Japan

\section{Taiichiro Kobayashi}

Department of Infectious Diseases, Tokyo Metropolitan Cancer and Infectious Diseases Center Komagome Hospital, Tokyo, Japan

\section{Tatsu Shimoyama}

Department of Medical Oncology, Tokyo Metropolitan Cancer and Infectious Diseases Center Komagome Hospital, Tokyo, Japan

\section{Yasushi Omuro}

Department of Medical Oncology, Tokyo Metropolitan Cancer and Infectious Diseases Center Komagome Hospital, Tokyo, Japan

\section{Noritaka Sekiya}

Department of Infection Prevention and Control, Department of Clinical Laboratory, Tokyo Metropolitan Cancer and Infectious Diseases Center Komagome Hospital, Tokyo, Japan 


\section{Akifumi Imamura}

Department of Infectious Diseases, Tokyo Metropolitan Cancer and Infectious Diseases Center Komagome Hospital, Tokyo, Japan

\section{Research Article}

Keywords: COVID-19, cancer, chemotherapy, lymphocytopenia, Japan

Posted Date: July 7th, 2020

DOl: https://doi.org/10.21203/rs.3.rs-39157/v1

License: (c) (1) This work is licensed under a Creative Commons Attribution 4.0 International License. Read Full License

Version of Record: A version of this preprint was published at International Journal of Clinical Oncology on November 23rd, 2020. See the published version at https://doi.org/10.1007/s10147-020-01837-0. 


\section{Abstract}

\section{Background}

Although severe acute respiratory syndrome coronavirus 2 (SARS-CoV-2) has caused an international outbreak of coronavirus disease 2019 (COVID-19), data on the clinical characteristics of COVID-19 patients with cancer are limited. This study aimed to evaluate the clinical characteristics and outcomes including mortality and viral shedding period in COVID-19 patients with cancer in Japan.

\section{Methods}

We retrospectively analyzed 32 patients with a history of cancer who were referred to our hospital between January 31, 2020 and May 25, 2020. We evaluated the association between clinical outcomes and potential prognostic factors using univariate analyses.

\section{Results}

The median age was 74.5 (range, 24-90) years and 22 patients (69\%) were men. A total of 11 patients (34\%) died. Our analyses demonstrated that the mortality was significantly associated with lymphocyte count, albumin, lactate dehydrogenase, serum ferritin, and C-reactive protein on admission. The median period between illness onset and the first effective negative SARS-CoV-2 PCR result was 22 days (interquartile range, 18-25) in survivors. Of four patients with hematological malignancy who developed COVID-19 within the rest period of chemotherapy, three died and the other patient, who received bendamustine plus rituximab therapy, had the longest duration of viral shedding ( 56 days).

\section{Conclusion}

Our study suggested that the risk factors for mortality previously reported in general COVID-19 patients, including lymphocytopenia, were also effective in cancer patients. Patients who received cytotoxic chemotherapy recently or were treated with chemotherapy, which can lead to lymphocyte reduction, had poor prognosis and prolonged periods of viral shedding.

\section{Introduction}

Coronavirus Disease 2019 (COVID-19), caused by severe acute respiratory syndrome coronavirus 2 (SARS-CoV-2), has spread globally since December 2019 [1,2]. In Japan, the first patient with COVID-19 was reported on January 15, 2020 [3], and the number of patients increased rapidly from March to May. In this context, COVID-19 has been a great concern for patients who have a history of cancer. Previous reports have shown that patients with cancer had a higher risk of developing severe events [4-12]. The World Health Organization-China Joint Mission reported that the overall mortality was $3.8 \%$, while the mortality of cancer patients was $7.6 \%$ in China [2]. 
Several studies reported the risk factors associated with development of severe events caused by CODIV19 in general people. A previous systematic review of 10 prognostic models for predicting poor prognosis in patients with COVID-19 showed that the most frequently reported predictors included age, sex, features derived from computed tomography (CT) scans, C-reactive protein (CRP), lactic dehydrogenase (LDH), and lymphocyte count [13]. Although some studies suggested the risk factors for mortality in cancer patients $[5,9]$, there are still insufficient data regarding the clinical characteristics and the risk factors for mortality in cancer patients. In particular, details of the viral shedding period and its association with chemotherapy have not been well described.

In this retrospective study, we evaluated the clinical characteristics and outcomes including the mortality and viral shedding period in COVID-19 patients with a history of cancer using the database in our hospital in Japan.

\section{Patients And Methods}

\section{Study design and patients}

This was a retrospective, single-center, observational study. We reviewed the records of 32 patients with a history of cancer out of 235 COVID-19 patients who were referred to our hospital between January 31, 2020 and May 25, 2020. Clinical data were retrieved from electronic medical records, including demographic and clinical features, laboratory findings, radiological data, and the results of reverse transcription polymerase chain reaction (RT-PCR) assay for SARS-CoV-2.

This study was approved by the institutional ethics review boards of our hospital, and the requirement to obtain a written informed consent was waived.

\section{Definitions}

All patients were diagnosed with COVID-19 by RT-PCR assay for SARS-CoV-2. The discharge criteria included symptom improvement and two consecutive negative results of RT-PCR test of nasopharyngeal swab taken at least 24 hours apart. The first effective negative PCR test was defined as the first test of the two negative PCR tests. The following comorbidities were also risk factors for COVID-19: diabetes, hypertension, coronary heart disease, chronic obstructive pulmonary disease (COPD), and asthma.

Patients with active cancer were defined as those who received treatment for cancer such as chemotherapy, immunotherapy, or hormone therapy within 30 days of COVID-19 onset, those scheduled to undergo cancer treatment, and/or those with metastasis to other organs. The illness onset was defined as the day when symptoms appeared.

\section{Statistical analysis}

We used the Mann-Whitney $U$ test or Fisher's exact test to compare the characteristics between survivors and non-survivors. We used a receiver operating characteristic curve in order to determine the best cut off value of laboratory data. Overall survival (OS) was defined as the period from illness onset to the date of 
last follow-up or death from any cause. OS was estimated using the Kaplan-Meier method. Differences between survival curves were tested for significance using the log-rank test. Two-sided $P$ values of 0.05 or less were considered significant. Hazard ratios and $95 \%$ confidence intervals were calculated with the use of Cox proportional-hazards models. All statistical analyses were performed with EZR (Saitama Medical Center, Jichi Medical University, Saitama, Japan), which is a graphical user interface for $\mathrm{R}$ (The R Foundation for Statistical Computing, Vienna, Austria). More precisely, it is a modified version of $\mathrm{R}$ commander designed to add statistical functions frequently used in biostatistics [14].

\section{Results}

\section{Patient characteristics}

A total of 235 patients were referred to our hospital with COVID-19 between January 31, 2020 and May 25,2020 . We retrospectively enrolled 32 patients with a history of cancer. They were all Asians except for one Australian. A total of 11 patients (34\%) eventually died, and all deaths were due to COVID-19. Twentyone patients $(66 \%)$ were discharged or transferred with negative RT-PCR results.

Table 1 shows the clinical characteristics of these patients. The median age was 74.5 (range, 24-90) years and 22 patients $(69 \%)$ were men. A total of 25 patients $(78 \%)$ had solid tumors, while $7(22 \%)$ had hematologic malignancies. In addition to cancer, 19 (59\%) had at least one comorbidity, with hypertension being the most common (13 patients, $41 \%)$. Thirteen patients $(41 \%)$ received cancer treatment within 30 days.

CT or X-ray on admission showed radiological features of pneumonia in 27 patients (84\%). Lymphocytopenia (lymphocyte count $<0.8 \times 10^{9} / \mathrm{L}$ ) occurred in 13 patients $(41 \%)$ and was predominant among the non-survivors ( $73 \%$ vs. $24 \%)$. Twenty-two patients $(69 \%)$ received favipiravir, while ten patients $(31 \%)$ were treated with systematic glucocorticoids.

The median time from illness onset to admission was 7 days (interquartile range [IQR], 4-8). The median time from illness onset to death was 24 days (IQR, 15-26). The median period between illness onset and the first effective negative SARS-CoV-2 RT-PCR result was 22 days (IQR, 18-25) in survivors.

\section{Risk factors for mortality}

The results of univariate analysis for OS are shown in Table 2. The following risk factors were significantly associated with mortality: lymphocyte count, albumin, LDH, serum ferritin, and CRP on admission. In our study, age, comorbidities, D-dimer, and cancer status were not associated with OS. The Kaplan-Meier survival curve stratified by high and low lymphocyte count is shown in Figure 1A (30-day OS: $90 \%$ and $46 \%, P=0.004)$. We further stratified patients with high and low lymphocyte count by comorbidities (30-day OS: $100 \%, 82 \%, 80 \%$, and $25 \%, P=0.003$, for high lymphocyte count and no comorbidities, high lymphocyte count and some comorbidities, low lymphocyte count and no comorbidities, and low lymphocyte count and some comorbidities) (Figure 1B). 
We suspected that the reduction in lymphocyte count was possibly caused by a certain factor, such as hematological toxicity of recent cytotoxic chemotherapy. Hence, we stratified patients with low lymphocyte count by whether they developed COVID-19 within the rest periods of cytotoxic chemotherapy or not. There was no significant difference between patients within the rest periods and other patients (30-day OS: $44 \%$ and $50 \%, P=0.75$ ) (Figure 1 C).

\section{Outcomes in patients with active cancer}

The clinical characteristics of 17 patients with active cancer are summarized in Supplementary Table 1. Seven patients received chemotherapy, while two patients received ICls (nivolumab and pembrolizumab + ipilimumab) within 30 days before the onset of COVID-19. Four patients received continuous hormone therapy. Pembrolizumab treatment was discontinued in one patient with metastatic lung cancer more than 200 days before the onset of COVID-19. One patient experienced a relapse with liver metastases after remission induced by repeated chemotherapy and surgery for rectal cancer. Two patients had not been treated yet as they developed COVID-19 before cancer treatment.

Figure 2 shows the timeline of cancer treatment, illness onset, SARS-CoV-2 RNA detection, and death in this subgroup of patients. The longest period between illness onset and the first effective negative SARSCoV-2 RT PCR result was 56 days in a patient who received bendamustine plus rituximab (BR) for Waldenström's macroglobulinemia/lymphoplasmacytic lymphoma. Moreover, another patient who received BR for mantle cell lymphoma 17 days before the illness onset tested positive for the virus at 42 days after the onset and eventually died. One patient who received azacytidine for acute myeloid leukemia (AML) 10 days before the onset and showed severe pancytopenia at the onset progressed rapidly and died in one week. One patient with ALK-negative anaplastic large cell lymphoma (ALCL) who received brentuximab vedotin, cyclophosphamide, doxorubicin, and prednisolone (A+CHP) 7 days prior to the onset of COVID-19 also died within 26 days. Among the four COVID-19 patients with hematological malignancy who developed COVID-19 within the rest periods of chemotherapy, three died and the other one had the longest duration of viral shedding among survivors. The two patients with AML and acute lymphocytic leukemia (ALL) had received induction and consolidation therapy, respectively. They both recovered from pancytopenia, remained in remission at the onset of COVID-19, and were eventually cured.

Two patients receiving ICls within 30 days eventually improved and were discharged, although one of them had elevated CRP and ferritin levels on admission and was treated with high-flow nasal cannula oxygen therapy without glucocorticoid therapy.

\section{Discussion}

To our knowledge, this is the first study to report on COVID-19 patients with a history of cancer in Japan. The factors associated with mortality were similar to those reported in previous studies conducted on general patients with COVID-19, including lymphocytopenia on admission. Patients who received myelosuppressive chemotherapy recently or received lymphocyte-reducing chemotherapy, such as BR, were found to have a greater risk of mortality and prolonged viral shedding periods. 
Lymphocytopenia is one of the frequently mentioned features of COVID-19 and correlates with clinical severity [15-17]. In the same way, T-cell count in peripheral blood is also significantly reduced and associated with high mortality rate [18-21]. Moreover, T-cells from COVID-19 patients have significantly higher expression levels of PD-1 and Tim-3, which suggests the surviving T-cell appear functionally exhausted [19]. These results imply that cellular immune response necessary for effective viral elimination is attenuated in COVID-19 patients. In our study, the detectable SARS-CoV-2 RNA persisted for a median of 22 days from the illness onset in survivors, which is almost the same as that reported in previous studies in general patients [22,23]. Interestingly, the virus was detectable for especially long duration in two patients who received $\mathrm{BR}$, one of whom eventually died. This treatment is known to suppress cellular immunity and reduce lymphocyte count strongly compared with rituximab plus $\mathrm{CHOP}$ (cyclophosphamide, doxorubicin, vincristine, and prednisolone) [24,25]. Thus, BR makes cellular immune response much weaker, thus leading to the prolongation of viral shedding and poor outcomes. Likewise, highly myelosuppressive chemotherapy for hematological malignancy causes pancytopenia, and also lymphocytopenia as a matter of course, and can lead to severe COVID-19. Our finding showed that patients with low lymphocyte count had poor OS regardless of the presence or absence of recent cytotoxic agent administration (Figure 1C). However, recent cytotoxic chemotherapy may be an important adverse factor in COVID-19 patients as a cause of lymphocytopenia.

Some retrospective study did not show any significant association between recent cancer treatment and mortality, as with our study [9-12]. However, one retrospective study in China reported a higher likelihood of experiencing severe events in patients who received antitumor treatment within 14 days of COVID-19 diagnosis [5]. In this study, two patients who received cytotoxic chemotherapy within the rest periods (azacytidine for AML and A+CHP for ALK-negative ALCL) developed severe COVID-19 and eventually died. On the contrary, patients with $A M L$ and $A L L$, who recovered from pancytopenia and remained in remission at the onset of COVID-19 after intensive treatment, were cured. Therefore, the risk of developing severe COVID-19 must be considered in patients who received myelosuppressive chemotherapy recently.

One of the important aggravating mechanisms of COVID-19 is cytokine release syndrome [26,27]. Meanwhile, immune checkpoint inhibitors may also activate the immune system and cause cytokine release syndrome [28]. One retrospective study reported that immunotherapy within 90 days was an independent risk factor for hospitalization and severe disease in cancer patients [10]. In our study, two patients received immunotherapy within 30 days. One patient had elevated CRP and ferritin levels on admission and required high-flow nasal cannula oxygen therapy. However, they eventually recovered even without receiving glucocorticoids. Further study is needed to confirm the association between immunotherapy and mortality of COVID-19.

Coagulopathy is also one of the mechanisms that can exacerbate COVID-19. Patients with COVID-19 are at risk of thromboembolism, and an increase in the concentrations of circulating D-dimer indicates pulmonary vascular bed thrombosis with fibrinolysis [29]. Several studies reported that elevated D-dimer on admission predicted mortality $[22,30,31]$. Meanwhile, cancer patients are originally at high risk of venous thromboembolism [32,33]. Although one retrospective cohort study in New York showed the 
association between D-dimer and mortality from COVID-19 in cancer patients [9], our study did not demonstrate a significant correlation between them. Determining the incidence of venous thrombosis in COVID-19 patients with cancer and interpreting the changes in D-dimer concentrations remain challenging.

This study has several limitations. First, this study was a retrospective, single center with a small sample size. Not all laboratory tests related to the prognosis of COVID-19, including albumin, serum ferritin, and D-dimer, were performed in all patients. We did not perform a multivariate analysis due to the lack of data and the small number of the study population. Second, the types of cancer and treatment were very heterogeneous in this study because we included all patients who had a history of cancer. Although it was difficult to draw a conclusive evidence from such a study population, our study provided new insight on the clinical features of COVID-19 patients with cancer. Future studies with a larger sample size are needed to further explore the risk factors for mortality in COVID-19 patients according to cancer or treatment types.

In conclusion, our study suggested that the risk factors for mortality previously reported in general COVID19 patients, including lymphocytopenia, were also effective in cancer patients. Awareness on the risk of chemotherapy that leads to severe cytopenia or suppresses cellular immunity during the COVID-19 pandemic must be improved.

\section{Declarations}

\section{Acknowledgments}

We would like to thank all the patients who took part in this study and Editage (www.editage.com) for English language editing.

\section{Funding}

No financial support was received for this study.

\section{Competing interests}

The authors declare no competing interests.

\section{References}

1. Zhu N, Zhang D, Wang W et al. (2020) A Novel Coronavirus from Patients with Pneumonia in China, 2019. The New England journal of medicine 382 (8):727-733. doi:10.1056/NEJMoa2001017

2. World Health Organization (2020) Report of the WHO-China Joint Mission on Coronavirus Disease 2019 (COVID-19). 16-24 February 2020. Available via DIALOG. https://www.who.int/docs/defaultsource/coronaviruse/who-china-joint-mission-on-covid-19-final-report.pdf. Accessed May 2020 
3. World Health Organization (2020) Novel Coronavirus (2019-nCoV): Situation Report - 1. 21 January 2020. Available via DIALOG. https://www.who.int/docs/default-source/coronaviruse/situationreports/20200121-sitrep-1-2019-ncov.pdf?sfvrsn=20a99c10_4. Accessed May 2020

4. Liang W, Guan W, Chen R et al. (2020) Cancer patients in SARS-CoV-2 infection: a nationwide analysis in China. The Lancet Oncology 21 (3):335-337. doi:10.1016/s1470-2045(20)30096-6

5. Zhang L, Zhu F, Xie L et al. (2020) Clinical characteristics of COVID-19-infected cancer patients: a retrospective case study in three hospitals within Wuhan, China. Annals of oncology : official journal of the European Society for Medical Oncology 31 (7):894-901. doi:10.1016/j.annonc.2020.03.296

6. Zhang HY, Wang LW, Chen YY et al. (2020) A Multicentre Study of 2019 Novel Coronavirus Disease Outcomes of Cancer Patients in Wuhan, China. medRxiv:2020.2003.2021.20037127. doi:10.1101/2020.03.21.20037127

7. He W, Chen L, Chen L et al. (2020) COVID-19 in persons with haematological cancers. Leukemia 34 (6):1637-1645. doi:10.1038/s41375-020-0836-7

8. Martín-Moro F, Marquet J, Piris M et al. (2020) Survival study of hospitalised patients with concurrent COVID-19 and haematological malignancies. British journal of haematology. doi:10.1111/bjh.16801

9. Mehta V, Goel S, Kabarriti R et al. (2020) Case Fatality Rate of Cancer Patients with COVID-19 in a New York Hospital System. Cancer Discov. doi:10.1158/2159-8290.cd-20-0516

10. Robilotti EV, Babady NE, Mead PA et al. (2020) Determinants of Severity in Cancer Patients with COVID-19 Illness. medRxiv. doi:10.1101/2020.05.04.20086322

11. Lee LYW, Cazier JB, Starkey T et al. (2020) COVID-19 mortality in patients with cancer on chemotherapy or other anticancer treatments: a prospective cohort study. Lancet (London, England). doi:10.1016/s0140-6736(20)31173-9

12. Kuderer NM, Choueiri TK, Shah DP et al. (2020) Clinical impact of COVID-19 on patients with cancer (CCC19): a cohort study. Lancet (London, England). doi:10.1016/s0140-6736(20)31187-9

13. Wynants L, Van Calster B, Collins GS et al. (2020) Prediction models for diagnosis and prognosis of covid-19 infection: systematic review and critical appraisal. BMJ (Clinical research ed) 369:m1328. doi:10.1136/bmj.m1328

14. Kanda Y (2013) Investigation of the freely available easy-to-use software 'EZR' for medical statistics. Bone marrow transplantation 48 (3):452-458. doi:10.1038/bmt.2012.244

15. Huang C, Wang Y, Li X et al. (2020) Clinical features of patients infected with 2019 novel coronavirus in Wuhan, China. Lancet (London, England) 395 (10223):497-506. doi:10.1016/s01406736(20)30183-5

16. Chen N, Zhou M, Dong X et al. (2020) Epidemiological and clinical characteristics of 99 cases of 2019 novel coronavirus pneumonia in Wuhan, China: a descriptive study. Lancet (London, England) 395 (10223):507-513. doi:10.1016/s0140-6736(20)30211-7

17. Yang X, Yu Y, Xu J et al. (2020) Clinical course and outcomes of critically ill patients with SARS-CoV2 pneumonia in Wuhan, China: a single-centered, retrospective, observational study. The Lancet Respiratory medicine 8 (5):475-481. doi:10.1016/s2213-2600(20)30079-5 
18. Diao B, Wang C, Tan Y et al. (2020) Reduction and Functional Exhaustion of T Cells in Patients With Coronavirus Disease 2019 (COVID-19). Frontiers in immunology 11:827.

doi:10.3389/fimmu.2020.00827

19. Jiang M, Guo Y, Luo Q et al. (2020) T cell subset counts in peripheral blood can be used as discriminatory biomarkers for diagnosis and severity prediction of COVID-19. The Journal of infectious diseases. doi:10.1093/infdis/jiaa252

20. Liu J, Li S, Liu J et al. (2020) Longitudinal characteristics of lymphocyte responses and cytokine profiles in the peripheral blood of SARS-CoV-2 infected patients. EBioMedicine 55:102763. doi:10.1016/j.ebiom.2020.102763

21. Wan S, Yi Q, Fan S et al. (2020) Characteristics of lymphocyte subsets and cytokines in peripheral blood of 123 hospitalized patients with 2019 novel coronavirus pneumonia (NCP). medRxiv:2020.2002.2010.20021832. doi:10.1101/2020.02.10.20021832

22. Zhou F, Yu T, Du R et al. (2020) Clinical course and risk factors for mortality of adult inpatients with COVID-19 in Wuhan, China: a retrospective cohort study. Lancet (London, England) 395 (10229):1054-1062. doi:10.1016/s0140-6736(20)30566-3

23. Xiao AT, Tong YX, Gao C et al. (2020) Dynamic profile of RT-PCR findings from 301 COVID-19 patients in Wuhan, China: A descriptive study. Journal of clinical virology : the official publication of the Pan American Society for Clinical Virology 127:104346. doi:10.1016/j.jcv.2020.104346

24. Rummel MJ, Niederle N, Maschmeyer G et al. (2013) Bendamustine plus rituximab versus CHOP plus rituximab as first-line treatment for patients with indolent and mantle-cell lymphomas: an open-label, multicentre, randomised, phase 3 non-inferiority trial. Lancet (London, England) 381 (9873):12031210. doi:10.1016/s0140-6736(12)61763-2

25. Flinn IW, van der Jagt R, Kahl BS et al. (2014) Randomized trial of bendamustine-rituximab or RCHOP/R-CVP in first-line treatment of indolent NHL or MCL: the BRIGHT study. Blood 123 (19):29442952. doi:10.1182/blood-2013-11-531327

26. Mehta P, McAuley DF, Brown M et al. (2020) COVID-19: consider cytokine storm syndromes and immunosuppression. Lancet (London, England) 395 (10229):1033-1034. doi:10.1016/s01406736(20)30628-0

27. Moore JB, June CH (2020) Cytokine release syndrome in severe COVID-19. Science (New York, NY) 368 (6490):473-474. doi:10.1126/science.abb8925

28. Rotz SJ, Leino D, Szabo S et al. (2017) Severe cytokine release syndrome in a patient receiving PD-1directed therapy. Pediatric blood \& cancer 64 (12). doi:10.1002/pbc.26642

29. McGonagle D, O'Donnell JS, Sharif K et al. (2020) Immune mechanisms of pulmonary intravascular coagulopathy in COVID-19 pneumonia. The Lancet Rheumatology. doi:10.1016/S26659913(20)30121-1

30. Wu C, Chen X, Cai Y et al. (2020) Risk Factors Associated With Acute Respiratory Distress Syndrome and Death in Patients With Coronavirus Disease 2019 Pneumonia in Wuhan, China. JAMA internal medicine. doi:10.1001/jamainternmed.2020.0994 
31. Zhang L, Yan X, Fan Q et al. (2020) D-dimer levels on admission to predict in-hospital mortality in patients with Covid-19. Journal of thrombosis and haemostasis : JTH 18 (6):1324-1329. doi: $10.1111 /$ jth. 14859

32. Walker AJ, Card TR, West J et al. (2013) Incidence of venous thromboembolism in patients with cancer - a cohort study using linked United Kingdom databases. European journal of cancer (Oxford, England : 1990) 49 (6):1404-1413. doi:10.1016/j.ejca.2012.10.021

33. Khorana AA, Kuderer NM, Culakova E et al. (2008) Development and validation of a predictive model for chemotherapy-associated thrombosis. Blood 111 (10):4902-4907. doi:10.1182/blood-2007-10116327

\section{Tables}

Table 1. Characteristics and outcomes of patients with a history of cancer 


\begin{tabular}{|c|c|c|c|c|}
\hline & Total $(n=32)$ & Survivors $(n=21)$ & Non-survivors $(n=11)$ & $P$ values \\
\hline \multicolumn{5}{|l|}{ al characteristics } \\
\hline ange), years & $74.5(24-90)$ & $72(24-87)$ & $76(53-90)$ & 0.38 \\
\hline$>70$ & $20(63 \%)$ & $12(57 \%)$ & $8(73 \%)$ & 0.47 \\
\hline $\operatorname{sex}$ & $22(69 \%)$ & $12(57 \%)$ & $10(91 \%)$ & 0.11 \\
\hline ng history (+) & $20 / 29(69 \%)$ & $14 / 19(74 \%)$ & $6 / 10(60 \%)$ & 0.67 \\
\hline omorbidities & $19(59 \%)$ & $10(48 \%)$ & $9(82 \%)$ & 0.13 \\
\hline betes & $7(22 \%)$ & $4(19 \%)$ & $3(27 \%)$ & 0.67 \\
\hline sertension & $13(41 \%)$ & $7(33 \%)$ & $6(55 \%)$ & 0.28 \\
\hline onary heart disease & $4(13 \%)$ & $2(10 \%)$ & $2(18 \%)$ & 0.59 \\
\hline onic obstructive lung disease & $4(13 \%)$ & $2(10 \%)$ & $2(18 \%)$ & 0.59 \\
\hline bma & $2(6 \%)$ & $1(5 \%)$ & $1(9 \%)$ & 1 \\
\hline$r$ diagnosis & & & & 0.67 \\
\hline natologic & $7(22 \%)$ & $4(19 \%)$ & $3(27 \%)$ & \\
\hline Acute myeloid leukemia & $2(6 \%)$ & $1(5 \%)$ & $1(9 \%)$ & \\
\hline Acute lymphocytic leukemia & $1(3 \%)$ & $1(5 \%)$ & 0 & \\
\hline Anaplastic large cell lymphoma & $1(3 \%)$ & 0 & $1(9 \%)$ & \\
\hline Mantle cell lymphoma & $1(3 \%)$ & 0 & $1(9 \%)$ & \\
\hline B-cell lymphoma & $1(3 \%)$ & $1(5 \%)$ & 0 & \\
\hline$W M / L P L$ & $1(3 \%)$ & $1(5 \%)$ & 0 & \\
\hline 1-hematologic & $25(78 \%)$ & $17(81 \%)$ & $8(73 \%)$ & \\
\hline Gastric cancer & $5(16 \%)$ & $4(19 \%)$ & $1(9 \%)$ & \\
\hline Colorectal cancer & $5(16 \%)$ & $4(19 \%)$ & $1(9 \%)$ & \\
\hline Prostatic cancer & $3(9 \%)$ & 0 & $3(27 \%)$ & \\
\hline Esophageal cancer & $2(6 \%)$ & $2(10 \%)$ & 0 & \\
\hline Lung cancer & $2(6 \%)$ & $1(5 \%)$ & $1(9 \%)$ & \\
\hline Breast cancer & $2(6 \%)$ & $2(10 \%)$ & 0 & \\
\hline Kidney cancer & $2(6 \%)$ & 0 & $2(18 \%)$ & \\
\hline Cervical cancer & $1(3 \%)$ & $1(5 \%)$ & 0 & \\
\hline Thymoma & $1(3 \%)$ & $1(5 \%)$ & 0 & \\
\hline Hepatocellular carcinoma & $1(3 \%)$ & $1(5 \%)$ & 0 & \\
\hline Skin cancer & $1(3 \%)$ & $1(5 \%)$ & 0 & \\
\hline \multicolumn{5}{|l|}{$r$ treatment } \\
\hline ration & $13(41 \%)$ & $9(43 \%)$ & $4(36 \%)$ & 1 \\
\hline mone therapy & $4(13 \%)$ & $2(10 \%)$ & $2(18 \%)$ & 0.59 \\
\hline motherapy & $10(31 \%)$ & $7(33 \%)$ & $3(27 \%)$ & 1 \\
\hline nunotherapy & $3(9 \%)$ & $2(10 \%)$ & $1(9 \%)$ & 1 \\
\hline motherapy/immunotherapy (within $\leq 30$ days) & $9(28 \%)$ & $6(29 \%)$ & $3(27 \%)$ & 1 \\
\hline treatment (within $\leq 30$ days) & $13(41 \%)$ & $8(38 \%)$ & $5(46 \%)$ & 0.72 \\
\hline$r$ status & & & & 1 \\
\hline ow-up/Cured & $12(43 \%)$ & $7(41 \%)$ & $5(46 \%)$ & \\
\hline ive cancer & $17(53 \%)$ & $11(52 \%)$ & $6(55 \%)$ & \\
\hline \multicolumn{5}{|l|}{$s$ and laboratory findings on admission } \\
\hline nonia on $C T$ or $X$-ray & $27(84 \%)$ & $16(76 \%)$ & $11(100 \%)$ & 0.14 \\
\hline blood cell count, $\times 10^{9} / \mathrm{L}$ & $5.6(3.6-6.7)$ & $5.4(3.6-6.3)$ & $5.7(3.6-12.7)$ & 0.38 \\
\hline ophil count, $\times 10^{9} / L$ & $3.6(2.4-4.5)$ & $3.5(2.4-4.1)$ & $4.0(2.2-11.3)$ & 0.33 \\
\hline 5 & $3(9 \%)$ & $1(5 \%)$ & $2(18 \%)$ & 0.23 \\
\hline hocyte count, $\times 10^{9} / L$ & $1.0(0.6-1.4)$ & $1.1(0.8-1.4)$ & $0.6(0.4-1.1)$ & 0.057 \\
\hline 8 & $13(41 \%)$ & $5(24 \%)$ & $8(73 \%)$ & 0.021 \\
\hline globin, $g / d L$ & $12.2(10.8-13.6)$ & $12.8(10.9-14.4)$ & $11.3(10.6-12.9)$ & 0.19 \\
\hline et count, $\times 10^{10} / \mathrm{L}$ & $20.5(12.0-24.7)$ & $21.2(17.5-24.9)$ & $15.3(8.1-19.3)$ & 0.074 \\
\hline inine, $m g / d L$ & $0.7(0.6-1.0)$ & $0.7(0.6-0.9)$ & $0.9(0.7-1.7)$ & 0.15 \\
\hline bilirubin, $m g / d L$ & $0.5(0.4-0.7)$ & $0.5(0.4-0.6)$ & $0.5(0.4-0.8)$ & 0.66 \\
\hline in, $g / L$ & $3.2(2.8-3.6)$ & $3.4(3.1-3.6)$ & $2.5(2.1-3.3)$ & 0.018 \\
\hline 0 & $8 / 27(30 \%)$ & $2 / 17(12 \%)$ & $6 / 10(60 \%)$ & 0.025 \\
\hline te dehydrogenase, $U / L$ & 260.5 (196.3-368.3) & $224(185.0-291.0)$ & 364 (246.0-418.5) & 0.068 \\
\hline 0 & $9(28 \%)$ & $3(14 \%)$ & $6(55 \%)$ & 0.035 \\
\hline
\end{tabular}




\begin{tabular}{|c|c|c|c|c|}
\hline 1 ferritin, $\mu g / L$ & $426.8(148.8-1104.0)$ & $280.5(124.1-686.4)$ & $1084.0(615.8-1894.0)$ & 0.029 \\
\hline 0 & $9 / 25(36 \%)$ & $4 / 18(22 \%)$ & $5 / 7(71 \%)$ & 0.058 \\
\hline stive protein, $\mathrm{mg} / \mathrm{dL}$ & $3.5(0.8-8.6)$ & $1.7(0.2-4.9)$ & $16.1(4.4-24.0)$ & 0.002 \\
\hline 0 & $13(41 \%)$ & $5(24 \%)$ & $8(73 \%)$ & 0.021 \\
\hline$e r, \mu g / L$ & $1.0(0.7-2.5)$ & $1.0(0.7-2.3)$ & $1.5(0.8-4.5)$ & 0.29 \\
\hline 0 & $13 / 26(50 \%)$ & $9 / 18(50 \%)$ & $4 / 8(50 \%)$ & 1 \\
\hline \multicolumn{5}{|l|}{ ments for COVID-19 } \\
\hline ravir & $22(69 \%)$ & $12(57 \%)$ & $10(91 \%)$ & 0.11 \\
\hline avir/ritonavir & $2(6 \%)$ & $1(5 \%)$ & $1(9 \%)$ & 1 \\
\hline onide & $5(16 \%)$ & $3(14 \%)$ & $2(18 \%)$ & 1 \\
\hline xychloroquine & $3(9 \%)$ & $1(5 \%)$ & $2(18 \%)$ & 0.27 \\
\hline matic glucocorticoids & $10(31 \%)$ & $3(14 \%)$ & $7(64 \%)$ & 0.013 \\
\hline \multicolumn{5}{|l|}{ mes } \\
\hline from onset to admission, days & $7(4-8)$ & $7(4-7)$ & $6(4-8)$ & 0.76 \\
\hline from onset to death, days & & & $24(15-26)$ & \\
\hline from onset to negative PCR result, days & & $22(18-25)$ & & \\
\hline
\end{tabular}

Data are expressed as n (\%), n/N (\%), or median (IQR), unless specified otherwise. WM/LPL, Waldenström's macroglobulinemia/lymphoplasmacytic lymphoma

Table 2. Univariable analysis for overall survivals

\begin{tabular}{lll}
\hline & & \\
\cline { 2 - 3 } & Univariate analysis & \\
\cline { 2 - 3 } Age $(>70)$ & $1.80(0.48-6.78)$ & 0.37 \\
Sex (male) & $4.82(0.62-37.73)$ & 0.093 \\
\hline Smoking history (+) & $0.55(0.15-1.96)$ & 0.34 \\
\hline Comorbidities (any) & $3.93(0.84-18.34)$ & 0.057 \\
\hline Diabetes & $1.60(0.42-6.07)$ & 0.48 \\
\hline Hypertension & $2.15(0.65-7.09)$ & 0.19 \\
\hline Coronary heart disease & $1.41(0.30-6.54)$ & 0.65 \\
\hline Chronic obstructive lung disease & $1.64(0.35-7.62)$ & 0.52 \\
\hline Asthma & $1.64(0.21-13.14)$ & 0.63 \\
\hline Cancer diagnosis $($ hematologic malignancy) & $1.36(0.36-5.13)$ & 0.64 \\
\hline Cancer treatment $($ any treatment within $\leq 30$ days) & $1.31(0.40-4.33)$ & 0.65 \\
\hline Cancer status $($ active cancer) & $1.16(0.35-3.83)$ & 0.80 \\
\hline Neutrophil count $(<1.5), \times 10^{9} / L$ & $2.73(0.59-12.67)$ & 0.18 \\
\hline Lymphocyte count $(<0.8), \times 10^{9} / L$ & $5.74(1.50-21.92)$ & 0.004 \\
\hline Albumin $(<3.0), g / L$ & $5.32(1.47-19.21)$ & 0.004 \\
\hline Lactate dehydrogenase $(>350), U / L$ & $4.40(1.33-14.63)$ & 0.008 \\
\hline Serum ferritin $(>800), \mu g / L$ & $6.12(1.18-31.79)$ & 0.014 \\
\hline C-reactive protein $(>5.0)$, mg/dL & $5.82(1.53-22.09)$ & 0.010 \\
\hline D-dimer $(>1.0), \mu g / L$ & $1.10(0.27-4.38)$ & 0.90 \\
\hline
\end{tabular}

CI, confidential interval 


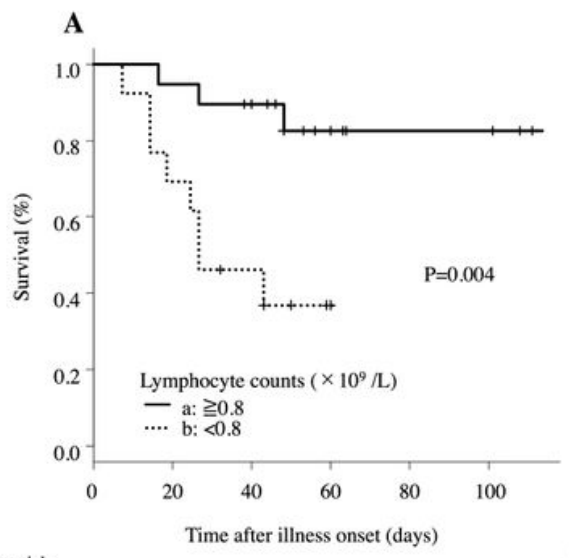

No. at risk

$\begin{array}{lcccccc}\text { a } & 19 & 18 & 16 & 7 & 4 & 4 \\ \text { b } & 13 & 9 & 5 & 1 & 0 & 0\end{array}$

B

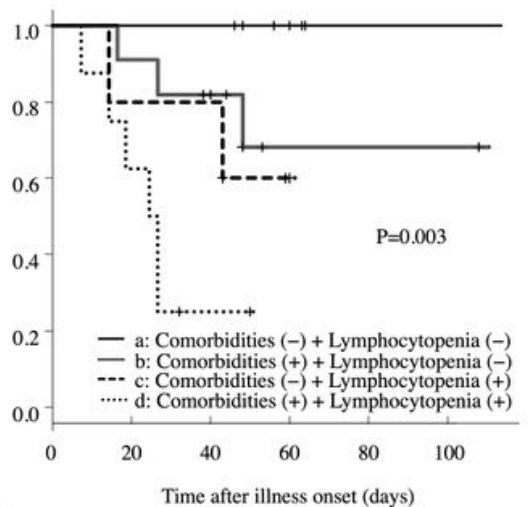

No. at risk

$\begin{array}{ll}\text { a } & 8 \\ \text { b } & 1 \\ \text { c } & 5 \\ \text { d } & 8\end{array}$
Time after illness onset (days)

$\begin{array}{ccccc}8 & 8 & 5 & 2 & 2 \\ 10 & 8 & 2 & 2 & 2 \\ 4 & 4 & 1 & 0 & 0 \\ 5 & 1 & 0 & 0 & 0\end{array}$

C (Patients with lymphocytopenia)

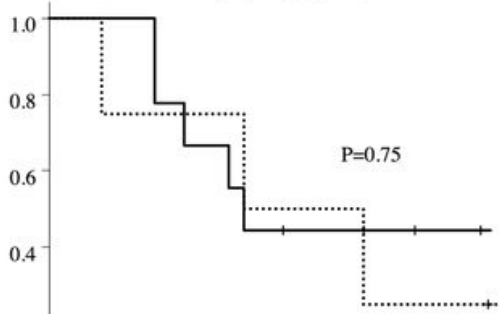

0.2 Develop COVID-19 within the rest periods of chemotherapy 一 a: No

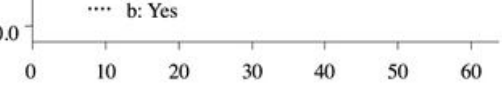

No. at risk

Time after illness onset (days)

\section{Figure 1}

Kaplan-Meier survival curves stratified by lymphocyte count, and lymphocyte count and comorbidities in all patients (A and B) and further stratified patients with low lymphocyte count by whether they developed COVID-19 within the rest periods of chemotherapy or not (C)

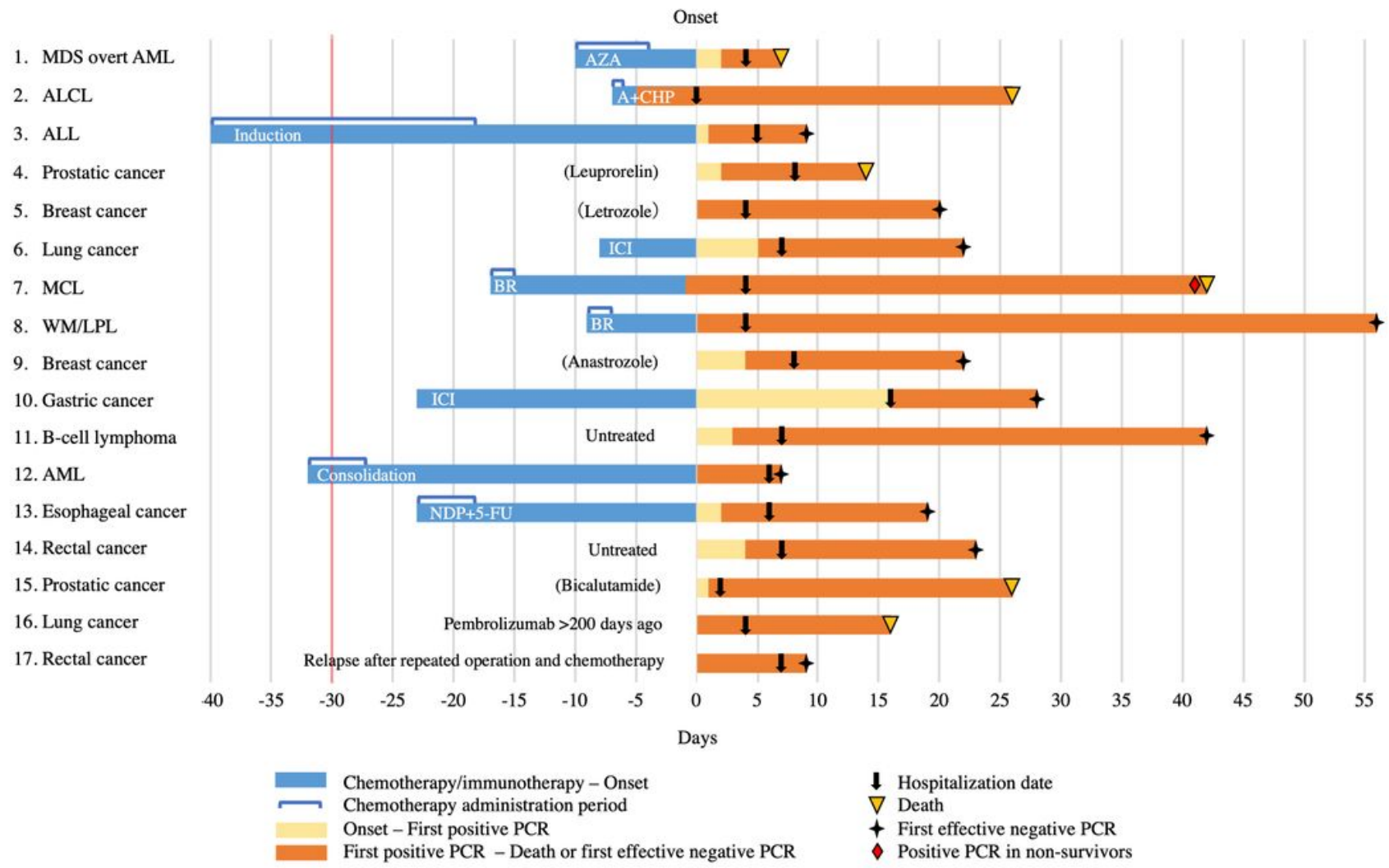

Figure 2 
Timeline of treatment for cancer, illness onset, SARS-CoV-2 RNA detection, and death in patients with active cancer. MDS, myelodysplastic syndromes; AML, acute myeloid leukemia; ALCL, anaplastic large cell lymphoma; $A L L$, acute lymphocytic leukemia; $M C L$, mantle cell lymphoma; WM/LPL, Waldenström's macroglobulinemia/lymphoplasmacytic lymphoma; $\mathrm{AZA}$, azacytidine; $\mathrm{A}+\mathrm{CHP}$, brentuximab vedotin, cyclophosphamide, doxorubicin, and prednisolone; ICl, immune checkpoint inhibitors, BR, bendamustine plus rituximab, NDP + 5-FU, nedaplatin and 5-fluorouracil

\section{Supplementary Files}

This is a list of supplementary files associated with this preprint. Click to download.

- supplementarytable1.docx 